\title{
Multi-dimensional Fejér summability and local Hardy spaces
}

by

\author{
FEREnC Weisz (Budapest)
}

\begin{abstract}
It is proved that the multi-dimensional maximal Fejér operator defined in a cone is bounded from the amalgam Hardy space $W\left(h_{p}, \ell_{\infty}\right)$ to $W\left(L_{p}, \ell_{\infty}\right)$. This implies the almost everywhere convergence of the Fejér means in a cone for all $f \in W\left(L_{1}, \ell_{\infty}\right)$, which is larger than $L_{1}\left(\mathbb{R}^{d}\right)$.
\end{abstract}

1. Introduction. It is known that the Fejér means $\sigma_{T} f$ of a function $f \in L_{1}(\mathbb{R})$ converge a.e. to $f$ as $T \rightarrow \infty$. Moreover, the maximal operator of the Fejér means $\sigma_{*}:=\sup _{T>0}\left|\sigma_{T}\right|$ is of weak type $(1,1)$, i.e.

$$
\sup _{\rho>0} \rho \lambda\left(\sigma_{*} f>\rho\right) \leq C\|f\|_{1} \quad\left(f \in L_{1}(\mathbb{R})\right)
$$

(see Zygmund [17, Vol. I. p. 154]). Móricz [9, 10] also verified that $\sigma_{*}$ is bounded from $H_{p}(\mathbb{R})$ to $L_{p}(\mathbb{R})$ for $p=1$. The author extended those results to all $1 / 2<p \leq 1$ in [14].

For multi-dimensional trigonometric-Fourier series Marcinkiewicz and Zygmund [8] proved that the Fejér means $\sigma_{n} f$ of a function $f \in L_{1}\left(\mathbb{T}^{d}\right)$ converge a.e. to $f$ as $n \rightarrow \infty$ provided that $n$ is in a positive cone, i.e., $2^{-\tau} \leq$ $n_{k} / n_{j} \leq 2^{\tau}$ for all $k, j=1, \ldots, d$ and some $\tau \geq 0\left(n=\left(n_{1}, \ldots, n_{d}\right) \in \mathbb{N}^{d}\right)$. The analogous convergence also holds for the Fejér means of Fourier transforms, i.e. $\sigma_{T} f \rightarrow f$ a.e. as $T \rightarrow \infty$ and $2^{-\tau} \leq T_{k} / T_{j} \leq 2^{\tau}(k, j=1, \ldots, d)$ for all $f \in L_{1}\left(\mathbb{R}^{d}\right)$ (see Weisz [15]). Moreover, the (restricted) maximal operator $\sigma_{*}$ defined in a cone is bounded from $H_{p}\left(\mathbb{R}^{d}\right)$ to $L_{p}\left(\mathbb{R}^{d}\right)$ whenever $d /(d+1)<p<\infty$.

In this paper we will prove sharper inequalities and convergence results. Goldberg [4] has introduced and investigated the so-called local Hardy spaces $h_{p}\left(\mathbb{R}^{d}\right)$. We will show that $\sigma_{*}$ is bounded from $h_{p}\left(\mathbb{R}^{d}\right)$ to $L_{p}\left(\mathbb{R}^{d}\right)$

2000 Mathematics Subject Classification: Primary 42B08, 46E30; Secondary 42B30, 42A38. Key words and phrases: Wiener amalgam spaces, local Hardy spaces, Fejér summability, Fourier transforms, atomic decomposition. 
$(d /(d+1)<p<\infty)$. This extends the results just mentioned, because the norm of $h_{p}\left(\mathbb{R}^{d}\right)$ is smaller than the one of $H_{p}\left(\mathbb{R}^{d}\right)$.

Starting with the local Hardy spaces we introduce new Wiener amalgam spaces $W\left(h_{p}, \ell_{\infty}\right)\left(\mathbb{R}^{d}\right)$. Our main result is that $\sigma_{*}$ is bounded from the amalgam space $W\left(h_{p}, \ell_{\infty}\right)\left(\mathbb{R}^{d}\right)$ to $W\left(L_{p}, \ell_{\infty}\right)\left(\mathbb{R}^{d}\right)(d /(d+1)<p<\infty)$ and from $W\left(L_{1}, \ell_{\infty}\right)\left(\mathbb{R}^{d}\right)$ to the weak space $W\left(L_{1, \infty}, \ell_{\infty}\right)\left(\mathbb{R}^{d}\right)$. By a density argument we show that the Fejér means $\sigma_{T} f$ converge a.e. to $f$ as $T \rightarrow \infty$ and $2^{-\tau} \leq T_{k} / T_{j} \leq 2^{\tau}(k, j=1, \ldots, d)$, provided that $f \in W\left(L_{1}, \ell_{\infty}\right)\left(\mathbb{R}^{d}\right)$. This is a significant generalization of the result for integrable functions, because $W\left(L_{1}, \ell_{\infty}\right)\left(\mathbb{R}^{d}\right)$ is a much larger space than $L_{1}\left(\mathbb{R}^{d}\right)$.

2. Local Hardy spaces and Wiener amalgams. Let us fix $d \geq 1$, $d \in \mathbb{N}$. Let $\lambda$ be the Lebesgue measure. We use the notation $|I|$ for the Lebesgue measure of the set $I$. For $x=\left(x_{1}, \ldots, x_{d}\right), u=\left(u_{1}, \ldots, u_{d}\right) \in \mathbb{R}^{d}$ we set $u \cdot x:=\sum_{k=1}^{d} u_{k} x_{k}$. We write briefly $L_{p}\left(\mathbb{R}^{d}\right)$ for the real $L_{p}\left(\mathbb{R}^{d}, \lambda\right)$ space with the norm (or quasinorm)

$$
\|f\|_{p}:=\left(\int_{\mathbb{R}^{d}}|f|^{p} d \lambda\right)^{1 / p} \quad(0<p \leq \infty) .
$$

The weak $L_{p}$ space, $L_{p, \infty}\left(\mathbb{R}^{d}\right)(0<p<\infty)$, consists of all measurable functions $f$ for which

$$
\|f\|_{L_{p, \infty}}:=\sup _{\rho>0} \rho \lambda(|f|>\rho)^{1 / p}<\infty .
$$

Note that $L_{p, \infty}\left(\mathbb{R}^{d}\right)$ is a quasi-normed space (see Bergh and Löfström $[1$, p. 8]). It is easy to see that for each $0<p \leq \infty$,

$$
L_{p}(\mathbb{R}) \subset L_{p, \infty}(\mathbb{R}) \quad \text { and } \quad\|\cdot\|_{L_{p, \infty}} \leq\|\cdot\|_{p} .
$$

The space of continuous functions with the supremum norm is denoted by $C(\mathbb{R})$, and $C_{c}(\mathbb{R})$ denotes the subspace of continuous functions having compact support.

For a measurable function $\phi$ on $\mathbb{R}^{d}$ let

$$
\phi_{t}(x):=t^{-d} \phi(x / t) \quad\left(x \in \mathbb{R}^{d}, t>0\right) .
$$

Given a Schwartz function $\phi \in \mathcal{S}\left(\mathbb{R}^{d}\right)$ with $\int_{\mathbb{R}^{d}} \phi d \lambda \neq 0$ and $\operatorname{supp} \phi \subset$ $[0,1 / 2]^{d}$, the local Hardy space $h_{p}\left(\mathbb{R}^{d}\right)(0<p \leq \infty)$ consists of all tempered distributions $f$ for which

$$
\|f\|_{h_{p}\left(\mathbb{R}^{d}\right)}:=\left\|\sup _{0<t<1}\left|f * \phi_{t}\right|\right\|_{L_{p}\left(\mathbb{R}^{d}\right)}<\infty .
$$

Replacing the $L_{p}\left(\mathbb{R}^{d}\right)$ norm by the $L_{p, \infty}\left(\mathbb{R}^{d}\right)$ norm, we get the weak local Hardy space $h_{p, \infty}\left(\mathbb{R}^{d}\right)(0<p \leq \infty)$. Taking the supremum over all $0<$ $t<\infty$ we obtain the definition of the classical Hardy space $H_{p}\left(\mathbb{R}^{d}\right)$. Other non-zero Schwartz functions $\phi$ define the same spaces and equivalent norms. 
Usually the classical Hardy spaces are investigated. The local Hardy spaces were introduced in Goldberg [4]. Using these spaces we will get convergence of Fejér sums for functions from $W\left(L_{1}, \ell_{\infty}\right)\left(\mathbb{R}^{d}\right)$, which is a much larger space than $L_{1}\left(\mathbb{R}^{d}\right)$.

It is known that the Hardy spaces $h_{p}\left(\mathbb{R}^{d}\right)$ and $H_{p}\left(\mathbb{R}^{d}\right)$ are equivalent to the $L_{p}\left(\mathbb{R}^{d}\right)$ space when $1<p \leq \infty$, and $H_{1}\left(\mathbb{R}^{d}\right) \subset h_{1}\left(\mathbb{R}^{d}\right) \subset L_{1}\left(\mathbb{R}^{d}\right)$. Moreover,

$$
\|f\|_{h_{1, \infty}} \leq\|f\|_{H_{1, \infty}} \leq C\|f\|_{1} \quad\left(f \in L_{1}\left(\mathbb{R}^{d}\right)\right)
$$

(see e.g. Stein [12, p. 91], Weisz [16, p. 68] and Goldberg [4]).

The atomic decomposition is a useful characterization of Hardy spaces. A bounded function $a$ is an $h_{p}\left(\mathbb{R}^{d}\right)$-atom if there exists a cube $I \subset \mathbb{R}^{d}$ such that

(i) $\operatorname{supp} a \subset I$,

(ii) $\|a\|_{\infty} \leq|I|^{-1 / p}$,

(iii) if $|I|<1$ then $\int_{I} a(x) x^{k} d \lambda(x)=0$ for all multi-indices $k=\left(k_{1}, \ldots, k_{d}\right)$ with $|k| \leq M$, where $M \geq[d(1 / p-1)]$ and $[x]$ denotes the integer part of $x \in \mathbb{R}$.

We will say that $a$ is a type 1 atom if $|I|<1$ and a type 2 atom if $|I| \geq 1$. If we require the moment condition in (iii) for all $|I| \geq 1$ then we obtain the definition of $H_{p}\left(\mathbb{R}^{d}\right)$-atom (see Lu [7, Chapter 1], Stein [12, Chapter 3] and Goldberg [4]).

Theorem 1. A tempered distribution $f$ is in $h_{p}\left(\mathbb{R}^{d}\right)(0<p \leq 1)$ if and only if there exist a sequence $\left(a_{k}, k \in \mathbb{N}\right)$ of $h_{p}\left(\mathbb{R}^{d}\right)$-atoms and a sequence $\left(\mu_{k}, k \in \mathbb{N}\right)$ of real numbers such that

$$
\sum_{k=0}^{\infty}\left|\mu_{k}\right|^{p}<\infty \text { and } \sum_{k=0}^{\infty} \mu_{k} a_{k}=f \quad \text { in the sense of distributions. }
$$

Moreover,

$$
\|f\|_{h_{p}} \sim \inf \left(\sum_{k=0}^{\infty}\left|\mu_{k}\right|^{p}\right)^{1 / p}
$$

where the infimum is taken over all decompositions of $f$ of the form (2).

Given a (quasi-)Banach space $X$ on $\mathbb{R}^{d}$, a measurable function $f$ belongs to the Wiener amalgam space $W\left(X, \ell_{q}\right)\left(\mathbb{R}^{d}\right)(0<q \leq \infty)$ if

$$
\|f\|_{W\left(X, \ell_{q}\right)}:=\left(\sum_{k \in \mathbb{Z}^{d}}\left\|\left.f\right|_{[k, k+1)}\right\|_{X}^{q}\right)^{1 / q}<\infty
$$

with the obvious modification for $q=\infty$, where $k+1:=\left(k_{1}+1, \ldots, k_{d}+1\right)$. $W\left(X, c_{0}\right)\left(\mathbb{R}^{d}\right)$ is defined analogously, where $c_{0}$ denotes the set of sequences with limit 0 . In this paper we will use the Wiener amalgam spaces for $X=$ 
$L_{p}\left(\mathbb{R}^{d}\right), L_{p, \infty}\left(\mathbb{R}^{d}\right), h_{p}\left(\mathbb{R}^{d}\right), h_{p, \infty}\left(\mathbb{R}^{d}\right)$. The closed subspace of $W\left(L_{\infty}, \ell_{q}\right)\left(\mathbb{R}^{d}\right)$ containing continuous functions is denoted by $W\left(C, \ell_{q}\right)\left(\mathbb{R}^{d}\right)(1 \leq q \leq \infty)$. The space $W\left(C, \ell_{1}\right)\left(\mathbb{R}^{d}\right)$ is called the Wiener algebra. It is used quite often in Gabor analysis, because it provides a convenient and general class of windows (see e.g. Gröchenig and Heil [5]). As can be seen in Feichtinger and Weisz $[2,3]$, it also plays an important role in summability theory. It is easy to see that

$$
W\left(L_{\infty}, \ell_{1}\right)\left(\mathbb{R}^{d}\right) \subset L_{p}\left(\mathbb{R}^{d}\right) \subset W\left(L_{1}, \ell_{\infty}\right)\left(\mathbb{R}^{d}\right) \quad(1 \leq p \leq \infty)
$$

and $W\left(L_{p}, \ell_{p}\right)\left(\mathbb{R}^{d}\right)=L_{p}\left(\mathbb{R}^{d}\right)$.

3. Fejér means of Fourier transforms. The Fourier transform of $f \in L_{1}\left(\mathbb{R}^{d}\right)$ is

$$
\hat{f}(x):=\int_{\mathbb{R}^{d}} f(t) e^{-2 \pi \imath x \cdot t} d t \quad\left(x \in \mathbb{R}^{d}\right),
$$

where $\imath=\sqrt{-1}$. If $f \in L_{p}\left(\mathbb{R}^{d}\right)$ for some $1 \leq p \leq 2$ then the Fourier inversion formula

$$
f(x)=\int_{\mathbb{R}^{d}} \hat{f}(u) e^{2 \pi \imath x \cdot u} d u \quad\left(x \in \mathbb{R}^{d}\right)
$$

holds if $\hat{f} \in L_{1}\left(\mathbb{R}^{d}\right)$. This motivates the definition of the Dirichlet integral $s_{t} f$ :

$$
s_{t} f(x):=\int_{-t_{1}}^{t_{1}} \ldots \int_{-t_{d}}^{t_{d}} \hat{f}(u) e^{2 \pi \imath x \cdot u} d u \quad\left(t=\left(t_{1}, \ldots, t_{d}\right) \in \mathbb{R}_{+}^{d}\right) .
$$

The Fejér means are defined by

$$
\sigma_{T} f(x):=\frac{1}{\prod_{j=1}^{d} T_{j}} \int_{0}^{T_{1}} \ldots \int_{0}^{T_{d}} s_{t} f(x) d t \quad\left(T=\left(T_{1}, \ldots, T_{d}\right) \in \mathbb{R}_{+}^{d}\right) .
$$

It is easy to see that

$$
\sigma_{T} f(x)=\int_{\mathbb{R}^{d}} f(t)\left(K_{T_{1}}\left(x_{1}-t_{1}\right) \times \cdots \times K_{T_{d}}\left(x_{d}-t_{d}\right)\right) d t
$$

where

$$
K_{S}(u):=S \operatorname{sinc}^{2}(S u):=S\left(\frac{\sin (\pi S u)}{\pi S u}\right)^{2} \quad(S>0, u \in \mathbb{R}, u \neq 0)
$$

is the Fejér kernel. Remark that

$$
\int_{\mathbb{R}} K_{S}(u) d u=\int_{\mathbb{R}} \operatorname{sinc}^{2}(u) d u=1 \quad(S>0)
$$

(see Zygmund [17, Vol. II, pp. 250-251]). 
The definition of the Fejér means can be extended easily from $L_{p}\left(\mathbb{R}^{d}\right)$ $(1 \leq p \leq 2)$ functions to all $f \in W\left(L_{1}, \ell_{\infty}\right)(\mathbb{R})$ by (3), i.e.

$$
\sigma_{T} f:=f *\left(K_{T_{1}} \times \cdots \times K_{T_{d}}\right) \quad\left(T \in \mathbb{R}_{+}^{d}\right) .
$$

For a fixed $\tau \geq 0$ the (restricted) maximal operator is defined by

$$
\sigma_{*} f:=\sup _{\substack{2^{-\tau} \leq T_{k} / T_{j} \leq 2^{\tau} \\ k, j=1, \ldots, d}}\left|\sigma_{T} f\right| .
$$

Equality (3) implies

$$
\left\|\sigma_{*} f\right\|_{\infty} \leq\left\|\operatorname{sinc}^{2}\right\|_{1}^{d}\|f\|_{\infty}=\|f\|_{\infty} \quad\left(f \in L_{\infty}(\mathbb{R})\right) .
$$

In this paper the constants $C$ and $C_{p}$ may vary from line to line, and the constants $C_{p}$ depend only on $p$.

4. Fejér summability and local Hardy spaces. In this section we generalize the results of Weisz [15] from $H_{p}\left(\mathbb{R}^{d}\right)$ spaces to $h_{p}\left(\mathbb{R}^{d}\right)$ and to the Wiener amalgam Hardy spaces.

Theorem 2. For $d /(d+1)<p<\infty$,

$$
\left\|\sigma_{*} f\right\|_{p} \leq C_{p}\|f\|_{h_{p}} \quad\left(f \in h_{p}\left(\mathbb{R}^{d}\right)\right)
$$

and

$$
\left\|\sigma_{*} f\right\|_{L_{1, \infty}} \leq C\|f\|_{1} \quad\left(f \in L_{1}\left(\mathbb{R}^{d}\right)\right) .
$$

Proof. For simplicity we prove the result for $d=2$ only. For $d>2$ the verification is very similar. Assume that $2 / 3<p \leq 1$ and $a$ is an arbitrary $h_{p}(\mathbb{R})$-atom with support $I$, where $I$ is a cube $I=I_{1} \times I_{2}$ and $2^{-K-1}<\left|I_{1}\right|=\left|I_{2}\right| \leq 2^{-K}$ for some $K \in \mathbb{Z}$. Let $4 I_{1}$ denote the interval with the same center as $I_{1}$ and with length $4\left|I_{1}\right|$, and $4 I:=4 I_{1} \times 4 I_{2}$. We may suppose that the center of $I$ is zero. If $a$ is a type 1 atom, i.e. $K \geq 0$, then

$$
\left\|\sigma_{*} a\right\|_{p} \leq C_{p}
$$

as proved in [15].

Now suppose that $a$ is a type 2 atom with $K<0$. By (5),

$$
\begin{aligned}
\int_{4 I}\left|\sigma_{*} a(x)\right|^{p} d x & \leq \int_{4 I} \sup _{T_{1}, T_{2} \geq 1}\left|\int_{\mathbb{R}^{2}} a(t) K_{T_{1}}\left(x_{1}-t_{1}\right) K_{T_{2}}\left(x_{2}-t_{2}\right) d t\right|^{p} d x \\
& \leq C_{p} 2^{2 K} 2^{-2 K} .
\end{aligned}
$$

Next we integrate over $\left(\mathbb{R} \backslash 4 I_{1}\right) \times 4 I_{2}$. We can see from (4) that

$$
\left|K_{S}(u)\right| \leq C S, \quad\left|K_{S}(u)\right| \leq \frac{C}{S|u|^{2}} \quad\left(u \in \mathbb{R} \backslash\{0\}, S \in \mathbb{R}_{+}\right) .
$$


Equality (3) implies that

$$
\left|\sigma_{T} a(x)\right| \leq C_{p} 2^{2 K / p} \int_{I_{1}} \frac{C}{T_{1}\left(x_{1}-t_{1}\right)^{2}} d t_{1} .
$$

We may suppose that $x_{i}>0(i=1,2)$. If $x_{1} \in\left[i 2^{-K},(i+1) 2^{-K}\right)(i \geq 1)$ then

$$
\left|\sigma_{*} a(x)\right| \leq C_{p} 2^{2 K / p} \int_{I_{1}} \frac{1}{\left(i 2^{-K}-2^{-K-1}\right)^{2}} d t_{1} \leq C_{p} 2^{K(2 / p+1)} i^{-2}
$$

and so

$$
\int_{\mathbb{R} \backslash 4 I_{1}} \int_{4 I_{2}}\left|\sigma_{*} a(x)\right|^{p} d x \leq \sum_{i=1}^{\infty} \int_{i 2^{-K}}^{(i+1) 2^{-K}} \int_{4 I_{2}}\left|\sigma_{*} a(x)\right|^{p} d x \leq C_{p} \sum_{i=1}^{\infty} 2^{K p} i^{-2 p},
$$

which is bounded, because $K<0$. The integral over $4 I_{1} \times\left(\mathbb{R} \backslash 4 I_{2}\right)$ can be estimated in the same way.

Similarly, if $x_{1} \in\left[i_{1} 2^{-K},\left(i_{1}+1\right) 2^{-K}\right)$ and $x_{2} \in\left[i_{2} 2^{-K},\left(i_{2}+1\right) 2^{-K}\right)$ $\left(i_{1}, i_{2} \geq 1\right)$ then

$$
\left|\sigma_{T} a(x)\right| \leq C_{p} 2^{2 K / p} \int_{I_{1}} \frac{C}{T_{1}\left(x_{1}-t_{1}\right)^{2}} d t_{1} \int_{I_{2}} \frac{C}{T_{2}\left(x_{2}-t_{2}\right)^{2}} d t_{2}
$$

and

$$
\left|\sigma_{*} a(x)\right| \leq C_{p} 2^{K(2 / p+2)} i_{1}^{-2} i_{2}^{-2}
$$

Thus

$$
\begin{aligned}
\int_{\mathbb{R} \backslash 4 I_{1}} \int_{\mathbb{R} \backslash 4 I_{2}}\left|\sigma_{*} a(x)\right|^{p} d x & \leq \sum_{i_{1}=1}^{\infty} \sum_{i_{2}=1}^{\infty} \int_{i_{1} 2^{-K}}^{\left(i_{1}+1\right) 2^{-K}} \int_{i_{2} 2^{-K}}^{\left(i_{2}+1\right) 2^{-K}}\left|\sigma_{*} a(x)\right|^{p} d x \\
& \leq C_{p} \sum_{i_{1}=1}^{\infty} \sum_{i_{2}=1}^{\infty} 2^{2 K p} i_{1}^{-2 p} i_{2}^{-2 p} \leq C_{p},
\end{aligned}
$$

which shows (7) for type 2 atoms as well as the theorem.

Recall that Theorem 2 was known for $H_{p}\left(\mathbb{R}^{d}\right)$ spaces (see Weisz [15]). The result for $h_{p}\left(\mathbb{R}^{d}\right)$ is stronger, because $\|\cdot\|_{h_{p}} \leq\|\cdot\|_{H_{p}}$. Next we show the boundedness of $\sigma_{*}$ on Wiener amalgam Hardy spaces.

Theorem 3. If $d /(d+1)<p<\infty$ then

$$
\left\|\sigma_{*} f\right\|_{W\left(L_{p}, \ell_{\infty}\right)} \leq C_{p}\|f\|_{W\left(h_{p}, \ell_{\infty}\right)} \quad\left(f \in W\left(h_{p}, \ell_{\infty}\right)\left(\mathbb{R}^{d}\right)\right)
$$

and

$$
\left\|\sigma_{*} f\right\|_{W\left(L_{1, \infty}, \ell_{\infty}\right)} \leq C_{p}\|f\|_{W\left(L_{1}, \ell_{\infty}\right)} \quad\left(f \in W\left(L_{1}, \ell_{\infty}\right)\left(\mathbb{R}^{d}\right)\right) .
$$


Proof. We are going to prove the result again for $d=2$ only. The proof is similar for higher dimensions. Let $2 / 3<p \leq 1$ and $\left.f\right|_{[j, j+1)}=\sum_{l=0}^{\infty} \mu_{j, l} a_{j, l}$ be an atomic decomposition of $\left.f\right|_{[j, j+1)} \in h_{p}\left(\mathbb{R}^{2}\right)$ such that

$$
\sum_{l=0}^{\infty}\left|\mu_{j, l}\right|^{p} \leq C_{p}\left\|\left.f\right|_{[j, j+1)}\right\|_{h_{p}}^{p} \leq C_{p}\|f\|_{W\left(h_{p}, \ell_{\infty}\right)}^{p} .
$$

Since $\operatorname{supp} \phi \subset[0,1 / 2]^{2}$, it follows that $\left.\operatorname{supp} f\right|_{[j, j+1)} * \phi_{t} \subset[j, j+3 / 2)$, where $j \in \mathbb{Z}^{2}, 0<t<1$. So we may suppose that $\operatorname{supp} a_{j, l} \subset[j, j+3 / 2)$. Thus

$$
\begin{aligned}
\int_{[k, k+1)}\left|\sigma_{*} f(x)\right|^{p} d x & \leq \sum_{j \in \mathbb{Z}^{2}} \int_{[k, k+1)}\left|\sigma_{*}\left(\left.f\right|_{[j, j+1)}\right)(x)\right|^{p} d x \\
& \leq \sum_{j \in \mathbb{Z}^{2}} \sum_{l=0}^{\infty}\left|\mu_{j, l}\right|^{p} \int_{[k, k+1)}\left|\sigma_{*} a_{j, l}(x)\right|^{p} d x
\end{aligned}
$$

for each fixed $k \in \mathbb{Z}^{2}$. Denote one of the atoms $a_{j, l}$ by $a$ and suppose that it is supported in a cube $I=I_{1} \times I_{2}$ with $2^{-K-1}<\left|I_{1}\right|=\left|I_{2}\right| \leq 2^{-K}$ for some $K \in \mathbb{Z}$. Then $I \subset[j, j+3 / 2)$.

Throughout this proof we may assume that $j_{i} \geq k_{i}$ for $i=1,2$. In (11) we have to integrate over

$$
\begin{aligned}
{[k, k+1)=} & ([k, k+1) \cap 4 I) \cup\left([k, k+1) \cap\left(\mathbb{R} \backslash 4 I_{1}\right) \times 4 I_{2}\right) \\
& \cup\left([k, k+1) \cap 4 I_{1} \times\left(\mathbb{R} \backslash 4 I_{2}\right)\right) \\
& \cup\left([k, k+1) \cap\left(\mathbb{R} \backslash 4 I_{1}\right) \times\left(\mathbb{R} \backslash 4 I_{2}\right)\right) .
\end{aligned}
$$

We do this in four steps.

STEP 1: Integrating over $[k, k+1) \cap 4 I$. It is easy to see that this set is empty if $j_{1} \geq k_{1}+4$ or $j_{2} \geq k_{2}+4$. For $k_{i} \leq j_{i} \leq k_{i}+3(i=1,2)$ we have, by (5),

$\int_{[k, k+1) \cap 4 I}\left|\sigma_{*} a(x)\right|^{p} d x$

$$
\leq \int_{4 I} \sup _{T_{1}, T_{2} \geq 1}\left|\int_{\mathbb{R}^{2}} a(t) K_{T_{1}}\left(x_{1}-t_{1}\right) K_{T_{2}}\left(x_{2}-t_{2}\right) d t\right|^{p} d x \leq C_{p} 2^{2 K} 2^{-2 K}
$$

and so

$$
\begin{aligned}
\sum_{j \in \mathbb{Z}^{2}} \sum_{l=0}^{\infty}\left|\mu_{j, l}\right|^{p} \int_{[k, k+1) \cap 4 I}\left|\sigma_{*} a_{j, l}(x)\right|^{p} d x & \leq C_{p} \sum_{j_{1}=k_{1}}^{k_{1}+3} \sum_{j_{2}=k_{2}}^{k_{2}+3} \sum_{l=0}^{\infty}\left|\mu_{j, l}\right|^{p} \\
& \leq C_{p}\|f\|_{W\left(h_{p}, \ell_{\infty}\right)}^{p} .
\end{aligned}
$$


STEP 2: Integrating over $[k, k+1) \cap\left(\mathbb{R} \backslash 4 I_{1}\right) \times 4 I_{2}$. This set is empty if $j_{2} \geq k_{2}+4$. If $a$ is a type 1 atom then

$$
\int_{[k, k+1) \cap\left(\mathbb{R} \backslash 4 I_{1}\right) \times 4 I_{2}}\left|\sigma_{*} a(x)\right|^{p} d x \leq \int_{\left(\mathbb{R} \backslash 4 I_{1}\right) \times 4 I_{2}}\left|\sigma_{*} a(x)\right|^{p} d x \leq C_{p}
$$

by (7). If $a$ is a type 2 atom and $j_{1}=k_{1}, k_{1}+1$, then the left hand side is 0 , because $\left|I_{1}\right| \geq 1$ and so $[k, k+1) \cap\left(\mathbb{R} \backslash 4 I_{1}\right) \times 4 I_{2}$ is empty. Thus

$$
\begin{aligned}
& \sum_{j_{1}=k_{1}}^{k_{1}+1} \sum_{j_{2} \in \mathbb{Z}} \sum_{l=0}^{\infty}\left|\mu_{j, l}\right|^{p} \int_{[k, k+1)} \cap\left(\mathbb{R} \backslash 4 I_{1}\right) \times 4 I_{2} \\
& \leq C_{p} \sum_{j_{1}=k_{1}}^{k_{1}+1} \sum_{j_{2}=k_{2}}^{k_{2}+3} \sum_{l=0}^{\infty}\left|\sigma_{*} a_{j, l}\right|^{p} \leq C_{p}\|f\|_{W\left(h_{p}, \ell_{\infty}\right)}^{p} .
\end{aligned}
$$

Assume that $j_{1} \geq k_{1}+2$. If $a$ is a type 1 atom then let

$$
A_{1}\left(x_{1}, t_{2}\right):=\int_{-\infty}^{x_{1}} a\left(t_{1}, t_{2}\right) d t_{1}, \quad A\left(x_{1}, x_{2}\right):=\int_{-\infty}^{x_{2}} A_{1}\left(x_{1}, t_{2}\right) d t_{2}
$$

$\left(x \in \mathbb{R}^{2}\right)$. Observe that

$$
\left\|A_{1}\right\|_{\infty} \leq C_{p} 2^{-K(1-2 / p)}, \quad\|A\|_{\infty} \leq C_{p} 2^{-K(2-2 / p)} .
$$

Integrating by parts in $t_{1}$ we can see that

$$
\begin{aligned}
\sigma_{T} a(x)= & \int_{I_{1}} \int_{I_{2}} a(t) K_{T_{1}}\left(x_{1}-t_{1}\right) K_{T_{2}}\left(x_{2}-t_{2}\right) d t \\
= & \int_{I_{2}} A_{1}\left(\nu_{1}, t_{2}\right) K_{T_{1}}\left(x_{1}-\nu_{1}\right) K_{T_{2}}\left(x_{2}-t_{2}\right) d t_{2} \\
& +\iint_{I_{1}} A_{1}(t) K_{T_{1}}^{\prime}\left(x_{1}-t_{1}\right) K_{T_{2}}\left(x_{2}-t_{2}\right) d t \\
= & : B_{1, T}(x)+B_{2, T}(x),
\end{aligned}
$$

where $I_{i}=\left[\mu_{i}, \nu_{i}\right](i=1,2)$. Using (8) we conclude that

$$
\left|B_{1, T}(x)\right| \leq C_{p} 2^{-K(1-2 / p)} \frac{C}{T_{1}\left(x_{1}-\nu_{1}\right)^{2}} T_{2} 2^{-K} \leq C_{p} 2^{-K(2-2 / p)}\left(j_{1}-k_{1}\right)^{-2},
$$

because $x_{1} \in\left(k_{1}, k_{1}+1\right)$ and $\nu_{1} \in I_{1} \subset\left[j_{1}, j_{1}+3 / 2\right)$. It is easy to see that

$$
\left|K_{S}^{\prime}(u)\right| \leq C S^{2}, \quad\left|K_{S}^{\prime}(u)\right| \leq \frac{C}{|u|^{2}} \quad\left(u \in \mathbb{R} \backslash\{0\}, S \in \mathbb{R}_{+}\right) .
$$

This, (5) and (12) imply that

$$
\left|B_{2, T}(x)\right| \leq C_{p} 2^{-K(1-2 / p)} \int_{I_{1}} \frac{C}{\left(x_{1}-t_{1}\right)^{2}} d t_{1} \leq C_{p} 2^{-K(2-2 / p)}\left(j_{1}-k_{1}\right)^{-2} .
$$


Hence

$$
\begin{aligned}
\int_{[k, k+1) \cap\left(\mathbb{R} \backslash 4 I_{1}\right) \times 4 I_{2}}\left|\sigma_{*} a(x)\right|^{p} d x & \leq \int_{\left(k_{1}, k_{1}+1\right) \times 4 I_{2}}\left|\sigma_{*} a(x)\right|^{p} d x \\
& \leq C_{p} 2^{-K(2 p-2)}\left(j_{1}-k_{1}\right)^{-2 p} 2^{-K}
\end{aligned}
$$

and

$$
\begin{aligned}
& \sum_{j_{1}=k_{1}+2}^{\infty} \sum_{j_{2} \in \mathbb{Z}} \sum_{l=0}^{\infty}\left|\mu_{j, l}\right|^{p} \int_{[k, k+1) \cap\left(\mathbb{R} \backslash 4 I_{1}\right) \times 4 I_{2}}\left|\sigma_{*} a_{j, l}(x)\right|^{p} d x \\
& \leq C_{p} \sum_{j_{1}=k_{1}+2}^{\infty} \sum_{j_{2}=k_{2}}^{k_{2}+3} \sum_{l=0}^{\infty}\left|\mu_{j, l}\right|^{p} 2^{-K(2 p-1)}\left(j_{1}-k_{1}\right)^{-2 p} \leq C_{p}\|f\|_{W\left(h_{p}, \ell_{\infty}\right)}^{p} .
\end{aligned}
$$

Similarly, if $a$ is a type 2 atom then the first line of (13) yields

$$
\left|\sigma_{T} a(x)\right| \leq C_{p} 2^{2 K / p} \int_{I_{1}} \frac{C}{T_{1}\left(x_{1}-t_{1}\right)^{2}} d t_{1} \leq C_{p} 2^{K(2 / p-1)}\left(j_{1}-k_{1}\right)^{-2}
$$

and so

$$
\begin{aligned}
& \sum_{j_{1}=k_{1}+2}^{\infty} \sum_{j_{2} \in \mathbb{Z}} \sum_{l=0}^{\infty}\left|\mu_{j, l}\right|^{p} \int_{[k, k+1) \cap\left(\mathbb{R} \backslash 4 I_{1}\right) \times 4 I_{2}}\left|\sigma_{*} a_{j, l}(x)\right|^{p} d x \\
& \leq C_{p} \sum_{j_{1}=k_{1}+2}^{\infty} \sum_{j_{2}=k_{2}}^{k_{2}+3} \sum_{l=0}^{\infty}\left|\mu_{j, l}\right|^{p} 2^{K(1-p)}\left(j_{1}-k_{1}\right)^{-2 p} \leq C_{p}\|f\|_{W\left(h_{p}, \ell_{\infty}\right)}^{p}
\end{aligned}
$$

because $K \leq 0$.

STEP 3: Integrating over $[k, k+1) \cap 4 I_{1} \times\left(\mathbb{R} \backslash 4 I_{2}\right)$. This case can be handled similarly to Step 2.

STEP 4: Integrating over $[k, k+1) \cap\left(\mathbb{R} \backslash 4 I_{1}\right) \times\left(\mathbb{R} \backslash 4 I_{2}\right)$. First assume that $j_{i}=k_{i}, k_{i}+1(i=1,2)$. Similarly to Step 2, (7) implies

$$
\int_{[k, k+1) \cap\left(\mathbb{R} \backslash 4 I_{1}\right) \times\left(\mathbb{R} \backslash 4 I_{2}\right)}\left|\sigma_{*} a(x)\right|^{p} d x \leq \int_{\left(\mathbb{R} \backslash 4 I_{1}\right) \times\left(\mathbb{R} \backslash 4 I_{2}\right)}\left|\sigma_{*} a(x)\right|^{p} d x \leq C_{p}
$$

if $a$ is a type 1 atom. For a type 2 atom the left hand side is again 0 . Thus

$$
\begin{aligned}
& \sum_{j_{1}=k_{1}}^{k_{1}+1} \sum_{j_{2}=k_{2}}^{k_{2}+1} \sum_{l=0}^{\infty}\left|\mu_{j, l}\right|^{p} \int_{[k, k+1)} \cap\left(\mathbb{R} \backslash 4 I_{1}\right) \times\left(\mathbb{R} \backslash 4 I_{2}\right) \\
& \leq C_{p} \sum_{j_{1}=k_{1}}^{k_{1}+1} \sum_{j_{2}=k_{2}}^{k_{2}+1} \sum_{l=0}^{\infty}\left|\sigma_{j, l}\right|^{p} \leq C_{p}\|f\|_{W\left(h_{p}, \ell_{\infty}\right)}^{p} .
\end{aligned}
$$

Next suppose that $j_{1} \geq k_{1}+2$ and $j_{2}=k_{2}, k_{2}+1$. The left hand side is 0 as above if $a$ is a type 2 atom. For a type 1 atom we integrate by parts 
in (13) with respect to $t_{2}$ :

$$
\begin{aligned}
\sigma_{T} a(x)= & -\int_{I_{2}} A\left(\nu_{1}, t_{2}\right) K_{T_{1}}\left(x_{1}-\nu_{1}\right) K_{T_{2}}^{\prime}\left(x_{2}-t_{2}\right) d t_{2} \\
& +\int_{I_{1}} A\left(t_{1}, \nu_{2}\right) K_{T_{1}}^{\prime}\left(x_{1}-t_{1}\right) K_{T_{2}}\left(x_{2}-\nu_{2}\right) d t_{1} \\
& -\int_{I_{1}} \int_{I_{2}} A(t) K_{T_{1}}^{\prime}\left(x_{1}-t_{1}\right) K_{T_{2}}^{\prime}\left(x_{2}-t_{2}\right) d t \\
= & : C_{1, T}(x)+C_{2, T}(x)+C_{3, T}(x) .
\end{aligned}
$$

One can easily prove from the estimates in (8) that

$$
\left|K_{S}(t)\right| \leq \frac{C S^{2 \eta-1}}{|t|^{2(1-\eta)}} \quad\left(t \in \mathbb{R} \backslash\{0\}, S \in \mathbb{R}_{+}\right)
$$

where $0 \leq \eta \leq 1$ is arbitrary. Similarly, for $0 \leq \eta \leq 1$, (14) implies

$$
\left|K_{S}^{\prime}(t)\right| \leq \frac{C S^{2 \eta}}{|t|^{2(1-\eta)}} \quad\left(t \in \mathbb{R} \backslash\{0\}, S \in \mathbb{R}_{+}\right) .
$$

If $x_{1} \in(k, k+1)$ and $x_{2} \in\left(\left(\mu_{2}+\nu_{2}\right) / 2-(i+1) 2^{-K},\left(\mu_{2}+\nu_{2}\right) / 2-i 2^{-K}\right)$ $(i \geq 1)$ (the center of $I_{2}$ is $\left.\left(\mu_{2}+\nu_{2}\right) / 2\right)$ then use (15) and (16) with $\eta=1 / 4$ to obtain

$$
\begin{aligned}
\left|C_{1, T}(x)\right| & \leq C_{p} 2^{-K(2-2 / p)} \frac{C T_{1}^{2 \eta-1}}{\left(x_{1}-\nu_{1}\right)^{2(1-\eta)}} \int_{I_{2}} \frac{C T_{2}^{2 \eta}}{\left(x_{2}-t_{2}\right)^{2(1-\eta)}} d t_{2} \\
& \leq C_{p} 2^{-K(2-2 / p)}\left(j_{1}-k_{1}\right)^{-2(1-\eta)} 2^{2 K(1-\eta)} i^{-2(1-\eta)} 2^{-K} \\
& =C_{p} 2^{-K(3 / 2-2 / p)}\left(j_{1}-k_{1}\right)^{-3 / 2} i^{-3 / 2}
\end{aligned}
$$

and

$$
\begin{aligned}
\left|C_{2, T}(x)\right| & \leq C_{p} 2^{-K(2-2 / p)} \int_{I_{1}} \frac{C T_{1}^{2 \eta}}{\left(x_{1}-t_{1}\right)^{2(1-\eta)}} d t_{1} \frac{C T_{2}^{2 \eta-1}}{\left(x_{2}-\nu_{2}\right)^{2(1-\eta)}} \\
& =C_{p} 2^{-K(3 / 2-2 / p)}\left(j_{1}-k_{1}\right)^{-3 / 2} i^{-3 / 2} .
\end{aligned}
$$

Hence for $m=1,2$,

$$
\begin{aligned}
\sum_{j_{1}=k_{1}+2}^{\infty} \sum_{j_{2}=k_{2}}^{k_{2}+1} \sum_{l=0}^{\infty}\left|\mu_{j, l}\right|^{p} \int_{[k, k+1) \cap\left(\mathbb{R} \backslash 4 I_{1}\right) \times\left(\mathbb{R} \backslash 4 I_{2}\right)}\left|C_{m, T}(x)\right|^{p} d x \\
\leq \sum_{j_{1}=k_{1}+2}^{\infty} \sum_{j_{2}=k_{2}}^{k_{2}+1} \sum_{l=0}^{\infty}\left|\mu_{j, l}\right|^{p} \int_{\left[k_{1}, k_{1}+1\right) \times\left(\mathbb{R} \backslash 4 I_{2}\right)}\left|C_{m, T}(x)\right|^{p} d x
\end{aligned}
$$




$$
\begin{aligned}
& \leq \sum_{j_{1}=k_{1}+2}^{\infty} \sum_{j_{2}=k_{2}}^{k_{2}+1} \sum_{l=0}^{\infty}\left|\mu_{j, l}\right|^{p} \sum_{i=1}^{\infty} \int_{k_{1}}^{k_{1}+1} \int_{\left(\mu_{2}+\nu_{2}\right) / 2-(i+1) 2^{-K}}^{\left(\mu_{2}+\nu_{2}\right) / 2-i 2^{-K}}\left|C_{m, T}(x)\right|^{p} d x \\
& \leq C_{p} \sum_{j_{1}=k_{1}+2}^{\infty} \sum_{j_{2}=k_{2}}^{k_{2}+1} \sum_{l=0}^{\infty} \sum_{i=1}^{\infty}\left|\mu_{j, l}\right|^{p} 2^{-K(3 p / 2-2)}\left(j_{1}-k_{1}\right)^{-3 p / 2} i^{-3 p / 2} 2^{-K} \\
& \leq C_{p}\|f\|_{W\left(h_{p}, \ell_{\infty}\right)}^{p} .
\end{aligned}
$$

For the third term we have

$$
\begin{aligned}
\left|C_{3, T}(x)\right| & \leq C_{p} 2^{-K(2-2 / p)} \int_{I_{1}} \frac{C}{\left(x_{1}-t_{1}\right)^{2}} d t_{1} \int_{I_{2}} \frac{C}{\left(x_{2}-t_{2}\right)^{2}} d t_{2} \\
& \leq C_{p} 2^{-K(2-2 / p)} 2^{-K}\left(j_{1}-k_{1}\right)^{-2} 2^{2 K} i^{-2} 2^{-K} \\
& =C_{p} 2^{-K(2-2 / p)}\left(j_{1}-k_{1}\right)^{-2} i^{-2}
\end{aligned}
$$

and

$$
\begin{aligned}
& \sum_{j_{1}=k_{1}+2}^{\infty} \sum_{j_{2}=k_{2}}^{k_{2}+1} \sum_{l=0}^{\infty}\left|\mu_{j, l}\right|^{p} \int_{[k, k+1) \cap\left(\mathbb{R} \backslash 4 I_{1}\right) \times\left(\mathbb{R} \backslash 4 I_{2}\right)}\left|C_{3, T}(x)\right|^{p} d x \\
& \quad \leq \sum_{j_{1}=k_{1}+2}^{\infty} \sum_{j_{2}=k_{2}}^{k_{2}+1} \sum_{l=0}^{\infty}\left|\mu_{j, l}\right|^{p} \sum_{i=1}^{\infty} \int_{k_{1}}^{k_{1}+1} \int_{\left(\mu_{2}+\nu_{2}\right) / 2-(i+1) 2^{-K}}^{\left(\mu_{2}+\nu_{2}\right) / 2-i 2^{-K}}\left|C_{3, T}(x)\right|^{p} d x \\
& \leq C_{p} \sum_{j_{1}=k_{1}+2}^{\infty} \sum_{j_{2}=k_{2}}^{k_{2}+1} \sum_{l=0}^{\infty} \sum_{i=1}^{\infty}\left|\mu_{j, l}\right|^{p 2^{-K(2 p-2)}\left(j_{1}-k_{1}\right)^{-2 p} i^{-2 p} 2^{-K}} \\
& \leq C_{p}\|f\|_{W\left(h_{p}, \ell_{\infty}\right)}^{p} .
\end{aligned}
$$

The case $j_{1}=k_{1}, k_{1}+1$ and $j_{2} \geq k_{2}+2$ can be handled similarly.

Finally, we suppose that $j_{i} \geq k_{i}+2$ for $i=1,2$. If $a$ is a type 1 atom and $x \in(k, k+1)$ then

$$
\begin{aligned}
\left|C_{1, T}(x)\right| & \leq C_{p} 2^{-K(2-2 / p)} \frac{C}{T_{1}\left(x_{1}-\nu_{1}\right)^{2}} \int_{I_{2}} \frac{C}{\left(x_{2}-t_{2}\right)^{2}} d t_{2} \\
& \leq C_{p} 2^{-K(3-2 / p)}\left(j_{1}-k_{1}\right)^{-2}\left(j_{2}-k_{2}\right)^{-2}, \\
\left|C_{2, T}(x)\right| & \leq C_{p} 2^{-K(2-2 / p)} \int_{I_{1}} \frac{C}{\left(x_{1}-t_{1}\right)^{2}} d t_{1} \frac{C}{\left(x_{2}-\nu_{2}\right)^{2}} \\
& \leq C_{p} 2^{-K(3-2 / p)}\left(j_{1}-k_{1}\right)^{-2}\left(j_{2}-k_{2}\right)^{-2}
\end{aligned}
$$


and so

$$
\begin{aligned}
& \sum_{j_{1}=k_{1}+2}^{\infty} \sum_{j_{2}=k_{2}+2}^{\infty} \sum_{l=0}^{\infty}\left|\mu_{j, l}\right|^{p} \int_{[k, k+1) \cap\left(\mathbb{R} \backslash 4 I_{1}\right) \times\left(\mathbb{R} \backslash 4 I_{2}\right)}\left|C_{m, T}(x)\right|^{p} d x \\
& \leq C_{p} \sum_{j_{1}=k_{1}+2}^{\infty} \sum_{j_{2}=k_{2}+2}^{\infty} \sum_{l=0}^{\infty}\left|\mu_{j, l}\right|^{p} 2^{-K(3 p-2)}\left(j_{1}-k_{1}\right)^{-2 p}\left(j_{2}-k_{2}\right)^{-2 p} \\
& \leq C_{p}\|f\|_{W\left(h_{p}, \ell_{\infty}\right)}^{p}
\end{aligned}
$$

for $m=1,2$. Similarly,

$$
\begin{aligned}
\left|C_{3, T}(x)\right| & \leq C_{p} 2^{-K(2-2 / p)} \int_{I_{1}} \frac{C}{\left(x_{1}-t_{1}\right)^{2}} d t_{1} \int_{I_{2}} \frac{C}{\left(x_{2}-t_{2}\right)^{2}} d t_{2} \\
& \leq C_{p} 2^{-K(4-2 / p)}\left(j_{1}-k_{1}\right)^{-2}\left(j_{2}-k_{2}\right)^{-2}
\end{aligned}
$$

and

$$
\begin{aligned}
& \sum_{j_{1}=k_{1}+2}^{\infty} \sum_{j_{2}=k_{2}+2}^{\infty} \sum_{l=0}^{\infty}\left|\mu_{j, l}\right|^{p} \int_{[k, k+1) \cap\left(\mathbb{R} \backslash 4 I_{1}\right) \times\left(\mathbb{R} \backslash 4 I_{2}\right)}\left|C_{3, T}(x)\right|^{p} d x \\
& \quad \leq C_{p} \sum_{j_{1}=k_{1}+2}^{\infty} \sum_{j_{2}=k_{2}+2}^{\infty} \sum_{l=0}^{\infty}\left|\mu_{j, l}\right|^{p} 2^{-K(4 p-2)}\left(j_{1}-k_{1}\right)^{-2 p}\left(j_{2}-k_{2}\right)^{-2 p} \\
& \leq C_{p}\|f\|_{W\left(h_{p}, \ell_{\infty}\right)}^{p} .
\end{aligned}
$$

For a type 2 atom we can see from (13) that

$$
\begin{aligned}
\left|\sigma_{T} a(x)\right| & \leq C_{p} 2^{2 K / p} \int_{I_{1}} \frac{C}{\left(x_{1}-t_{1}\right)^{2}} d t_{1} \int_{I_{2}} \frac{C}{\left(x_{2}-t_{2}\right)^{2}} d t_{2} \\
& \leq C_{p} 2^{-K(2-2 / p)}\left(j_{1}-k_{1}\right)^{-2}\left(j_{2}-k_{2}\right)^{-2}
\end{aligned}
$$

and

$$
\begin{aligned}
& \sum_{j_{1}=k_{1}+2}^{\infty} \sum_{j_{2}=k_{2}+2}^{\infty} \sum_{l=0}^{\infty}\left|\mu_{j, l}\right|^{p} \int_{[k, k+1) \cap\left(\mathbb{R} \backslash 4 I_{1}\right) \times\left(\mathbb{R} \backslash 4 I_{2}\right)}\left|\sigma_{T} a(x)\right|^{p} d x \\
& \quad \leq C_{p} \sum_{j_{1}=k_{1}+2}^{\infty} \sum_{j_{2}=k_{2}+2}^{\infty} \sum_{l=0}^{\infty}\left|\mu_{j, l}\right|^{p} 2^{-K(2 p-2)}\left(j_{1}-k_{1}\right)^{-2 p}\left(j_{2}-k_{2}\right)^{-2 p} \\
& \leq C_{p}\|f\|_{W\left(h_{p}, \ell_{\infty}\right)}^{p} .
\end{aligned}
$$

Taking into account (11) we have thus finished the proof of (9) for $2 / 3<p \leq 1$.

It is known that $h_{\infty}\left(\mathbb{R}^{2}\right)=L_{\infty}\left(\mathbb{R}^{2}\right)$ (see e.g. Stein [12, p. 91]) and $W\left(L_{\infty}, \ell_{\infty}\right)\left(\mathbb{R}^{2}\right)=L_{\infty}\left(\mathbb{R}^{2}\right)$. The boundedness of $\sigma_{*}$ from $W\left(h_{\infty}, \ell_{\infty}\right)\left(\mathbb{R}^{2}\right)$ to $W\left(L_{\infty}, \ell_{\infty}\right)\left(\mathbb{R}^{2}\right)$ follows from (6). Applying this and (9) for $p=1$ we use 
complex interpolation to deduce that

$$
\begin{aligned}
\sigma_{*}:\left(W\left(h_{1}, \ell_{\infty}\right)\left(\mathbb{R}^{2}\right), W\left(h_{\infty}, \ell_{\infty}\right)\right. & \left.\left(\mathbb{R}^{2}\right)\right)_{[\eta]} \\
& \rightarrow\left(W\left(L_{1}, \ell_{\infty}\right)\left(\mathbb{R}^{2}\right), W\left(L_{\infty}, \ell_{\infty}\right)\left(\mathbb{R}^{2}\right)\right)_{[\eta]}
\end{aligned}
$$

is bounded, where $0<\eta<1$ is arbitrary. Using the method of Triebel $[13$, p. 121] we can prove that

$$
\left(W\left(h_{1}, \ell_{\infty}\right)\left(\mathbb{R}^{2}\right), W\left(h_{\infty}, \ell_{\infty}\right)\left(\mathbb{R}^{2}\right)\right)_{[\eta]}=W\left(\left(h_{1}, h_{\infty}\right)_{[\eta]}, \ell_{\infty}\right)\left(\mathbb{R}^{2}\right)
$$

and that the same holds with $L_{1}$ instead of $h_{1}$. Choosing $\eta=1-1 / p$ $(1<p<\infty)$ we obtain the boundedness of

$$
\sigma_{*}: W\left(h_{p}, \ell_{\infty}\right)\left(\mathbb{R}^{2}\right) \rightarrow W\left(L_{p}, \ell_{\infty}\right)\left(\mathbb{R}^{2}\right),
$$

which is exactly (9).

By the real method of interpolation we have

$$
\left(W\left(h_{p}, \ell_{\infty}\right)\left(\mathbb{R}^{2}\right), W\left(h_{\infty}, \ell_{\infty}\right)\left(\mathbb{R}^{2}\right)\right)_{\eta, \infty}=W\left(\left(h_{p}, h_{\infty}\right)_{\eta, \infty}, \ell_{\infty}\right)\left(\mathbb{R}^{2}\right)
$$

and the analogue for $L_{p}$ (see Sagher [11], Kisliakov and Xu [6], Berg and Löfström [1, Chapter 5]). We conclude that

$$
\sigma_{*}: W\left(\left(h_{p}, h_{\infty}\right)_{\eta, \infty}, \ell_{\infty}\right)\left(\mathbb{R}^{2}\right) \rightarrow W\left(\left(L_{p}, L_{\infty}\right)_{\eta, \infty}, \ell_{\infty}\right)\left(\mathbb{R}^{2}\right)
$$

is bounded, where $0<\eta<1$ is arbitrary. If $p<1$ then the choice $\eta=1-p$ implies the boundedness of

$$
\sigma_{*}: W\left(h_{1, \infty}, \ell_{\infty}\right)\left(\mathbb{R}^{2}\right) \rightarrow W\left(L_{1, \infty}, \ell_{\infty}\right)\left(\mathbb{R}^{2}\right)
$$

and inequality (1) proves (10). This completes the proof of Theorem 3.

REMARK 1. The exact value of $C_{p}$ in (10) is $C_{p}=C_{p}\left\|\operatorname{sinc}^{2}\right\|_{1}^{d(1-p)}$ because of (6) and the basic theorems of interpolation theory, where $1 / 2<$ $p<1$ is fixed.

Remark 1 will be used in the next corollary for $\left.\operatorname{sinc}^{2}\right|_{(-k, k)^{c}}$ instead of sinc $^{2}$. Since $W\left(L_{1}, \ell_{\infty}\right)\left(\mathbb{R}^{d}\right) \supset L_{1}\left(\mathbb{R}^{d}\right)$, the next corollary is much more general than the results in [14].

Corollary 1. For all $f \in W\left(L_{1}, \ell_{\infty}\right)\left(\mathbb{R}^{d}\right)$,

$$
\lim _{T \rightarrow \infty} \sigma_{T} f=f \quad \text { a.e. }
$$

as $\min \left(T_{1}, \ldots, T_{d}\right) \rightarrow \infty$ and $2^{-\tau} \leq T_{k} / T_{j} \leq 2^{\tau}(k, j=1, \ldots, d)$.

Proof. For $f \in C_{c}\left(\mathbb{R}^{2}\right)$ we obtain the convergence from

$$
\begin{aligned}
\left|\sigma_{T} f(x)-f(x)\right| & =\left|\sigma_{T} f(x)-\left(\int_{\mathbb{R}} \operatorname{sinc}^{2} d \lambda\right)^{2} f(x)\right| \\
& \leq \int_{\mathbb{R}^{2}}\left|f\left(x_{1}-\frac{t_{1}}{T_{1}}, x_{2}-\frac{t_{2}}{T_{2}}\right)-f(x)\right| \operatorname{sinc}^{2}\left(t_{1}\right) \operatorname{sinc}^{2}\left(t_{2}\right) d t
\end{aligned}
$$


and from the Lebesgue dominated convergence theorem. Since $C_{c}\left(\mathbb{R}^{2}\right)$ is dense in $W\left(L_{1}, c_{0}\right)\left(\mathbb{R}^{2}\right)$, the corollary follows for all $f \in W\left(L_{1}, c_{0}\right)\left(\mathbb{R}^{2}\right)$ from (10) and the usual density argument due to Marcinkiewicz and Zygmund [8]. Observe that Theorem 3 and Remark 1 can be applied for the functions $\left.\operatorname{sinc}^{2}\right|_{(-k, k)}(k \in \mathbb{N})$ instead of $\operatorname{sinc}^{2}$. Hence

$$
\lim _{T \rightarrow \infty, 2^{-\tau} \leq T_{k} / T_{j} \leq 2^{\tau}} \sigma_{T, \operatorname{sinc}^{2} \times\left.\operatorname{sinc}^{2}\right|_{(-k, k)^{2}}} f=\left(\left.\int_{\mathbb{R}} \operatorname{sinc}^{2}\right|_{(-k, k)} d \lambda\right)^{2} f \text { a.e. }
$$

for all $f \in W\left(L_{1}, c_{0}\right)\left(\mathbb{R}^{2}\right)$, where

$$
\sigma_{T, h_{1} \times h_{2}} f(x):=\int_{\mathbb{R}^{2}} f\left(x_{1}-\frac{t_{1}}{T_{1}}, x_{2}-\frac{t_{2}}{T_{2}}\right) h_{1}\left(t_{1}\right) h_{2}\left(t_{2}\right) d t .
$$

It is easy to see that if $x \in[-j, j]^{2}$ then for $T_{i} \geq 1(i=1,2)$,

$$
\sigma_{T, \operatorname{sinc}^{2} \times\left.\operatorname{sinc}^{2}\right|_{(-k, k)} ^{2}} f(x)=\sigma_{T, \operatorname{sinc}^{2} \times\left.\operatorname{sinc}^{2}\right|_{(-k, k)^{2}}}\left(\left.f\right|_{[-j-k, j+k]^{2}}\right)(x) .
$$

However, $\left.f\right|_{[-j-k, j+k]^{2}} \in W\left(L_{1}, c_{0}\right)\left(\mathbb{R}^{2}\right)$ if $f \in W\left(L_{1}, \ell_{\infty}\right)\left(\mathbb{R}^{2}\right)$, and so (18) holds for all $f \in W\left(L_{1}, \ell_{\infty}\right)\left(\mathbb{R}^{2}\right)$. Fix $f \in W\left(L_{1}, \ell_{\infty}\right)\left(\mathbb{R}^{2}\right)$ and set

$$
\xi:=\limsup _{T \rightarrow \infty, 2^{-\tau} \leq T_{k} / T_{j} \leq 2^{\tau}}\left|\sigma_{T} f-f\right| .
$$

We have (with the same meaning of lim sup)

$$
\begin{aligned}
& \xi \leq \lim \sup \left|\sigma_{T} f-\sigma_{T, \operatorname{sinc}^{2} \times\left.\operatorname{sinc}^{2}\right|_{(-k, k)} ^{2}} f\right| \\
& +\lim \sup \left|\sigma_{T, \operatorname{sinc}^{2} \times\left.\operatorname{sinc}^{2}\right|_{(-k, k)} ^{2}} f-\left(\int_{\mathbb{R}^{2}} \operatorname{sinc}^{2} \times\left.\operatorname{sinc}^{2}\right|_{(-k, k)^{2}} d \lambda\right) f\right| \\
& +\left|\left(\int_{\mathbb{R}^{2}} \operatorname{sinc}^{2} \times\left.\operatorname{sinc}^{2}\right|_{(-k, k)^{2}} d \lambda\right)^{2} f-f\right| \\
& \leq \lim \sup \left|\sigma_{T, \operatorname{sinc}^{2} \times\left.\operatorname{sinc}^{2}\right|_{\mathbb{R}^{2} \backslash(-k, k)^{2}}} f\right|+\left(\int_{\mathbb{R}^{2} \backslash(-k, k)^{2}} \operatorname{sinc}^{2} \times \operatorname{sinc}^{2} d \lambda\right)|f|
\end{aligned}
$$

for all $k \in \mathbb{N}$. By Theorem 3 and Remark 1 we conclude that

$$
\begin{aligned}
\|\xi\|_{W\left(L_{1, \infty}, \ell_{\infty}\right)} \leq & \| \sup _{T \geq 1,2^{-\tau} \leq T_{k} / T_{j} \leq 2^{\tau}} \mid \sigma_{T, \operatorname{sinc}^{2} \times\left.\operatorname{sinc}^{2}\right|_{\mathbb{R}^{2} \backslash(-k, k)^{2}} f \mid \|_{W\left(L_{1, \infty}, \ell_{\infty}\right)}} \\
& +\left(\int_{\mathbb{R}^{2} \backslash(-k, k)^{2}} \operatorname{sinc}^{2} \times \operatorname{sinc}^{2} d \lambda\right)\|f\|_{W\left(L_{1, \infty}, \ell_{\infty}\right)} \\
\leq & C_{p}\left\|\operatorname{sinc}^{2} \times\left.\operatorname{sinc}^{2}\right|_{\mathbb{R}^{2} \backslash(-k, k)^{2}}\right\|_{1}^{1-p}\|f\|_{W\left(L_{1}, \ell_{\infty}\right)} \\
& +\left\|\operatorname{sinc}^{2} \times\left.\operatorname{sinc}^{2}\right|_{\mathbb{R}^{2} \backslash(-k, k)^{2}}\right\|_{1}\|f\|_{W\left(L_{1}, \ell_{\infty}\right)}
\end{aligned}
$$

for all $k \in \mathbb{N}$, where $2 / 3<p<1$. Since $\left\|\operatorname{sinc}^{2} \times\left.\operatorname{sinc}^{2}\right|_{\mathbb{R}^{2} \backslash(-k, k)^{2}}\right\|_{1} \rightarrow 0$ as $k \rightarrow \infty,\|\xi\|_{W\left(L_{1, \infty}, \ell_{\infty}\right)}=0$ and so $\xi=0$ a.e., which finishes the proof. 
Acknowledgments. This research was supported by the Hungarian Scientific Research Funds (OTKA) No K67642.

\section{References}

[1] J. Bergh and J. Löfström, Interpolation Spaces, an Introduction, Springer, Berlin, 1976.

[2] H. G. Feichtinger and F. Weisz, The Segal algebra $\mathbf{S}_{0}\left(\mathbb{R}^{d}\right)$ and norm summability of Fourier series and Fourier transforms, Monatsh. Math. 148 (2006), 333-349.

[3] - - - Wiener amalgams and pointwise summability of Fourier transforms and Fourier series, Math. Proc. Cambridge Philos. Soc. 140 (2006), 509-536.

[4] D. Goldberg, A local version of real Hardy spaces, Duke Math. J. 46 (1979), 27-42.

[5] K. Gröchenig and C. Heil, Gabor meets Littlewood-Paley: Gabor expansions in $L^{p}\left(\mathbb{R}^{d}\right)$, Studia Math. 146 (2001), 15-33.

[6] S. V. Kisliakov and Q. Xu, Interpolation of weighted and vector-valued Hardy spaces, Trans. Amer. Math. Soc. 343 (1994), 1-34.

[7] S. Lu, Four Lectures on Real $H^{p}$ Spaces, World Sci., Singapore, 1995.

[8] J. Marcinkiewicz and A. Zygmund, On the summability of double Fourier series, Fund. Math. 32 (1939), 122-132.

[9] F. Móricz, The maximal Fejér operator for Fourier transforms of functions in Hardy spaces, Acta Sci. Math. (Szeged) 62 (1996), 537-555.

[10] - The maximal Fejér operator is bounded from $H^{1}(\mathbf{T})$ to $L^{1}(\mathbf{T})$, Analysis 16 (1996), 125-135.

[11] Y. Sagher, Interpolation of r-Banach spaces, Studia Math. 16 (1972), 45-70.

[12] E. M. Stein, Harmonic Analysis: Real-Variable Methods, Orthogonality, and Oscillatory Integrals, Princeton Univ. Press, Princeton, NJ, 1993.

[13] H. Triebel, Interpolation Theory, Function Spaces, Differential Operators, Johann Ambrosius Barth Verlag, Heidelberg, 1995.

[14] F. Weisz, The maximal Fejér operator of Fourier transforms, Acta Sci. Math. (Szeged) 64 (1998), 447-457.

[15] - The maximal Fejér operator of multi-dimensional Fourier transforms, East J. Approx. 4 (1998), 491-503.

[16] - Summability of Multi-Dimensional Fourier Series and Hardy Spaces, Math. Appl. 541, Kluwer, Dordrecht, 2002.

[17] A. Zygmund, Trigonometric Series, 3rd ed., Cambridge Univ. Press, London, 2002.

Department of Numerical Analysis

Eötvös L. University

Pázmány P. sétány 1/C

H-1117 Budapest, Hungary

E-mail: weisz@numanal.inf.elte.hu 